\title{
Whitening Dentifrices: A Review
}

\author{
Ceren Değer' (1), Arzu Müjdeci² (1) \\ 'Department of Restorative Dentistry, Bezmialem Vakıf University School of Dentistry, İstanbul, Turkey \\ 2Department of Restorative Dentistry, Ankara University School of Dentistry, Ankara, Turkey
}

ORCID iDs of the authors: C.D. 0000-0002-2100-8963; A.M. 0000-000I-8272-5782.

Cite this article as: Değer C, Müjdeci A. Whitening Dentifrices: A Review. Cyprus J Med Sci 2020; 5(4): 355-60.

\begin{abstract}
Whitening dentifrices are used in dental procedures for achieving aesthetic effects, such as tooth cleaning and stain removal. Today, owing to the effect of media, there is an increasing interest among individuals to have a healthy and beautiful smile, resulting in higher use of easily accessible whitening dentifrices. The whitening effect of whitening dentifrices is mostly attributed to their constituent abrasives or chemical, optical, or enzymatic ingredients. Thus, it is important to have updated knowledge regarding the ingredients, whitening efficacy, and mechanisms regarding the action of whitening dentifrices. Several dentifrices are available in the global market, and dentists should be aware that the selection of the most appropriate dentifrice is possible with correct knowledge about the content of each dentifrice. Based on their ingredients, certain whitening dentifrices may negatively affect dental hard tissues. Thus, dentists should warn patients about the potential adverse effects of whitening dentifrices on teeth and the negative consequences of dentifrices with unproven whitening claims.
\end{abstract}

Keywords: Whitening dentifrices, abrasive, blue covarine, charcoal, activated carbon

\section{INTRODUCTION}

The color of a tooth is a combination of the internal color and external pigmentations that may present on the tooth surface. The internal color of the tooth is affected by the absorption and reflection properties of the dentine and enamel. However, the most important determinant of the overall tooth color is the dentin structure (I). Tooth discoloration can be classified as extrinsic or intrinsic as per the location of the stain. Extrinsic stains are present on the tooth surface or in the acquired pellicle. They can be consequences of smoking habit; poor brushing technique; the use of certain cationic agents, such as chlorhexidine; and/or a diet rich in colored foods or drinks, such as coffee, tea, and red wine. Intrinsic discolorations occur with changes in the structural composition or thickness of dental hard tissues. Systemic factors, such as excessive fluoride intake, erythroblastosis fetalis, and systemic medication, can cause internal discolorations (2).

Owing to the influence of media, patients are showing an increasing interest in getting whiter and brighter teeth. Various methods have been developed to resolve discoloration and make teeth look whiter to fulfill patient requirements. These methods include the use of whitening dentifrices, enamel microabrasion, vital bleaching as home/office, non-vital bleaching, and prosthetic treatments (3).

Dentist-supervised home bleaching technique is the most commonly used whitening procedure (4). In this treatment, $10 \%$ carbamide peroxide gel is placed in a tray, and patients apply the tray themselves at certain hours during the day for a period of $2 \mathrm{wk}$. The benefits of the home bleaching technique and patient satisfaction have encouraged the marketing of over-the-counter products (5). Gels, rinses, whitening strips, and dentifrices are some examples of the over-the-counter whitening products (3).

Whitening dentifrices with their ease of use and low cost have been getting increasing attention from consumers $(I)$ and are perhaps the most accessible product type for many patients and consumers who want to whiten their teeth (6). Different whitening dentifrices that have different mechanisms of action are available in the market. Whitening dentifrices are generally formulated to remove external stains using physical methods and to prevent the reformation of these discolorations (7). Today, it is widely accepted that the primary mechanism effective in whitening dentifrices is the abrasive mechanism although other ingredients, including chemical agents, enzymes, and optic agents are added into these dentifrices to remove stains and prevent them from re-appearing $(\mathrm{I})$. 


\section{Mechanism of Action and Ingredients of Whitening Dentifrices}

\begin{abstract}
Abrasives
Abrasives added as insoluble particles in toothpastes have been used for tooth cleaning in combination with toothbrush bristles for about 2000 y (7). Abrasives are physically harder than the stain (I), and they remove stained dental plaque on tooth surface mechanically. Although abrasives constitute the main mechanism in the elimination and prevention of external stains, they are only effective on external stains and reach only those areas that can be accessed using a toothbrush. Moreover, they have limited effects in malocclusion regions, interdental areas, and gingival areas (I), and they do not change the color of the teeth.
\end{abstract}

Whitening dentifrices containing abrasives have been formulated with specifically designed higher content of abrasives than that in standard dentifrices (2). It is important for whitening dentifrices to have maximal cleaning and stain-removing effect with minimal hard tissue wear (8). The stain removal effect of whitening dentifrices that contain abrasives depends on the abrasive particle size, shape, distribution, concentration (9), as well as $\mathrm{pH}$ of the dentifrices (10). When the abrasive particles are too large, the toothbrush bristles are unable to catch these particles and the particles are swept away from the tooth surface and become ineffective (I). The hard tissue wear effect of the dentifrices and the relative safety of abrasivity have been standardized for enamel and dentine as radioactive dentin abrasion (RDA) and radioactive enamel abrasion methods. These methods enable a comparison of the abrasiveness of dentifrices and evaluate their appropriate lifetime use by patients (II). The International Organization for Standardization (ISO) has determined the abrasivity limit for dentifrices as 250 RDA (12). Whitening dentifrices having RDA 60-100 are considered to have medium abrasiveness and those with RDA>100 are considered highly abrasive (II).

Whitening dentifrices can contain the abrasives as monoabrasives or a mixture of different abrasives. Abrasives having chemically different types may exert varying cleaning effectiveness; chemically similar abrasives may have different cleaning ratios. Moreover, the mixture of chemically different abrasives can have a different effect than individual abrasive components (I3). The following abrasives are used in whitening dentrifices: hydrated silica, calcium carbonate, dicalcium phosphate dihydrate, calcium pyrophosphate, alumina, perlite, and sodium bicarbonate $(\mathrm{I}, 2)$.

\section{Main Points:}

- Chemical and mechanical substances have been added to whitening dentifrices to increase the effect of abrasive cleaning in areas that cannot be reached by a toothbrush and to ensure the effectiveness of dentifrice.

- Dentifrices containing proteolytic enzymes are good alternatives for patients with dentin hypersensitivity to dentifrices containing abrasives that cause enamel and dentin wear.

- Today, many dentifrices are sold on the market for whitening. Dentists should be aware of the ingredients in these dentifrices, recommend which is right for their patients, and warn about possible side effects.
Silica abrasive technology has generally been used in dentifrices. The most common abrasives are hydrated silica and calcium carbonate. Hydrated silica is a gentle abrasive that has the ability to clean teeth stains very effectively (I3). It is compatible with most active ingredients in dentifrices, and the Mohs' scale hardness of hydrated silica is 5 (14).

A type of silica abrasive, "soft silica" abrasive, has been recently introduced and has reduced Mohs' scale hardness (conventional silica=5.9, soft silica=4.2) and dentine abrasion (conventional silica RDA=\|0, soft silica RDA=87). When soft silica is added into a toothpaste formulation, in vitro studies have showed a significant improvement in the pellicle-cleaning ratio than that with conventional silica formulations. This result has been further supported in a clinical study on chlorhexidine/tea-induced stain removal and a natural stain prevention study (I5).

Another type of abrasive, high cleaning silica that is not abrasive on teeth and provides maximum stain removal effect has also been incorporated into whitening dentifrices (16). Significant natural stain removal with a high cleaning silica dentifrice was found comparable to that with silica-containing dentifrice over a period of $6 \mathrm{wk}(17)$.

Perlite is an amorphous glassy silicate used as a polishing agent in prophylaxis products that are associated with excellent stain removal and low abrasivity. Perlite added into a silica-based dentifrice or a calcium carbonate dentifrice has shown significant improvement in stain removal as compared to a control silica dentifrice in both, in vitro and in vivo studies $(18,19)$.

Sodium bicarbonate has low abrasivity and Mohs' scale hardness of 2.5; moreover, it is compatible with active ingredients in dentifrices (14). The whitening effect of toothpastes containing sodium bicarbonate in a high concentration is greater than that of toothpastes containing silica and calcium phosphate. This result has been explained by the low abrasiveness, low internal hardness, and high solubility of calcium phosphate. Dentifrices containing sodium bicarbonate in different concentrations have been found in vitro to be more effective in removing intrinsic yellow stains than water or classical dentifrices (20). Koertge ef al. (20) have examined the whitening effect of a classic dentifrice and a dentifrice containing $65 \%$ sodium bicarbonate in 115 volunteers over a period of for $12 \mathrm{wk}$. The dentifrice containing sodium bicarbonate was significantly more effective in reducing the discoloration and increasing the whiteness compared to a classic dentifrice. Yankell et al. (2I) have found that sodium bicarbonate/calcium peroxide containing dentifrices are more effective than silica-based tartar dentifrices for controlling extrinsic stains. Bollmer et al. (22) have investigated the effect of 3.3\% pyrophosphate containing tartar control dentifrice and classic dentifrice containing hydrated silica and disodium pyrophosphate on chlorhexidine induced stain formation in vivo. They have observed that both the dentifrices significantly reduced the coloration on the buccal surfaces of the anterior teeth.

\section{Chemical Agents}

It is difficult to remove intrinsic stains with dentifrices that remove stains mechanically with abrasive ingredients. Therefore, various chemical agents that have non-abrading whitening effect have been added into whitening dentifrices (I). 
The following chemical agents are used in whitening dentifrices: hydrogen peroxide, calcium peroxide, sodium citrate, sodium pyrophosphate, sodium tripolyphosphate, sodium hexametaphosphate, and sodium chlorite (I).

Hydrogen peroxide is used as a tooth whitening agent for about 100 y and was first used in 1989 when Haywood and Heymann introduced home-type bleaching. Today, peroxides are added into dentifrices to oxidize intrinsic stain molecules and change their absorption spectrum to lower visibility and bleach the enamel. The American Dental Association has stated that hydrogen peroxide concentrations of $\geq 3 \%$ should not be considered for frequent or extended use owing to the risk of damage to oral tissues (23). Therefore, in dentifrices, the concentration of peroxides is very low (usually hydrogen peroxide $1 \%$, calcium peroxide $0.5 \%-0.7 \%$ ), and the percentage of hydrogen peroxide allowed in Europe is only 0.1\%. A low concentration of peroxide is permitted; thus, the difficulty in incorporation of peroxide into dentifrice and short application time can limit the effectiveness of peroxide containing dentifrices in removing intrinsic stains (I, 14). Therefore, both, chemical and mechanical agents have been added into whitening dentifrices to contribute to the effect of abrasive cleaning and ensure the effectiveness of the dentifrice in areas that cannot be reached with a toothbrush (I).

A dentifrice containing $1 \%$ hydrogen peroxide as a chemical agent has shown a significant reduction in the extrinsic stains as compared to toothpastes containing only silica and silica/hexametaphosphate in vitro (23). Hydrogen peroxide in whitening dentifrices has interfered with chromatic alterations of the teeth within 2-6 weeks of brushing (24). Kleber et al. (25) have compared a $1 \%$ hydrogen peroxide/sodium bicarbonate containing toothpaste to a toothpaste with silica/sodium bicarbonate. They reported that $1 \%$ hydrogen peroxide/sodium bicarbonate containing toothpaste was more effective in reducing yellowness and increasing brightness. In an in vivo study with whitening dentifrices containing hydrogen peroxide in the range of $1 \%$ concentration, an improvement was noted in the tooth color (24). No superiority of whitening dentifrices with chemical whitening agents has been reported. A whitening dentifrice containing hydrogen peroxide, calcium pyrophosphate, and tetrapotassium phosphate as chemical agents has shown similar extrinsic stain removal performance as compared to an ordinary dentifrice in a study by Soares et al. (26).

Chemical agents, such as pyrophosphate, tripolyphosphate, and hexametaphosphate have binding affinity to enamel, dentin, and tartar. They remove stain components during adsorption and prevent the formation of new pigments. Pyrophosphates dislocate anions and negatively charged macromolecules that are present in the acquired enamel pellicle to remove stains (27). Sodium pyrophosphate is frequently used in whitening dentifrices in addition to its use in tartar removal. Dentifrices containing sodium tripolyphosphate and pyrophosphate combination are more effective than non-bleaching silica dentifrices containing only sodium tripolyphosphate (27).

Sodium hexametaphosphate, another chemical agent, is a longchain pyrophosphate variant. It attaches to the surface of the teeth with multiple bonding zones, and when compared to pyrophosphate, it prevents the formation of tea/coffee-induced stains (28).

\section{Enzymes}

Extrinsic stains are primarily incorporated into the pellicle. Enzymes, such as proteases, that break down proteins can be effective on the protein portion of the pellicle and can be used to remove extrinsic stains without abrasive actions (I). Therefore, whitening toothpastes that contain natural enzyme extracts derived from plants have been manufactured as non-abrading whitening agents (29). Nowadays, enzymes incorporated into the dentifrices for whitening purposes are usually natural proteolytic enzymes, such as papain and bromelain, that belong to a group of proteases (30). Papain is derived from the plant Carica Papaya, and Bromelain is derived from Ananas Camosus (3I). Both the enzymes hydrolyze and break the protein pellicles down on the tooth surface, thus inhibiting the attachment of oral microorganisms and stain on the tooth surface (32). An enzymatic dentifrice with papain and bromelain has been found to be more effective for stain removal than control dentifrice in vitro (33). Moreover, this enzyme-containing dentifrice has shown considerable residual bleaching effect even after at the end of the 2 months owing to its proteolytic and antibacterial actions (29). In clinical studies, the efficacy of enzymatic dentifrices has been reported, and a dentifrice containing papain, alumina, and citrate has shown to reduce discolorations (34).

Dentifrices containing proteolytic enzymes that do not contain abrasive ingredients are reported to be a good alternative for patients with dentin hypersensitivity instead of dentifrices containing abrasives causing enamel and dentin wear (29). However, the shelf life of enzymatic dentifrices is shorter than that of regular dentifrices owing to their proteolytic structure (3I).

\section{Optical Agents}

Perception of tooth color is a complex phenomenon that is influenced by the experience of the person evaluating the tooth color, the illumination of the environment, the presence of internal or external colorations, and the color of the lips. Commission International De L'Eclairage (CIE) introduced a three-dimensional mathematical color system (CIELAB) for identifying color perception in 1976. This system consists of the following 3 axis: $L^{*}, a^{*}$ and $b^{*} ; L^{*}$ represents lightness of the object, where $a^{*}$ represents red-green scale and $b^{*}$ represents the yellow-blue scale (35).

The use of an optical principle is an alternative approach to tooth whitening. Decrease in $b^{*}$ value causes color change from yellow to blue tone and makes the patient perceive his/ her own teeth as whiter (36). Considering that the deposition of blue colored agents onto the tooth surface causes a shift in the color from yellow to blue, a blue pigment like blue covarine has been added into dentifrices. Blue covarine pigment simulates the scattering wavelengths of the enamel in the blue extension and changes the appearance from yellowish to bluish. Blue covarine adds a thin blue layer to the enamel surface and alters the perception of the tooth color; thus, a silica-based whitening dentifrice containing blue covarine has been developed. Dentifrices containing blue covarine cause a sudden and significant reduction in tooth yellowness and enhance the whiteness (37).

A study that compared dentifrices containing blue covarine in different concentrations and a dentifrice without blue covarine has showed that brushing once with the dentifrice containing blue covarine could increase the tooth whiteness compared to 
the control dentifrice. In addition, as the concentration of blue covarine increased, tooth yellowness decreased with the decrease in $b^{*}$ values, and the whiteness increased (37).

Some whitening dentifrices contain another optical agent, titanium dioxide, a white pigment. Titanium dioxide attaches into surface irregularities, resulting in a whiter tooth illusion as only a surface phenomenon instead of internal color change (38).

\section{Other Ingredients}

Some agents, such as detergents and antimicrobials, that are included in regular toothpastes, can enhance the whitening effect.

Dentifrices containing sodium lauryl sulfate reduce the surface tension of the stain molecules and prevent the binding of these molecules to the enamel (7). Some plaque bacteria are reported to have chromogenic properties that cause green, brown, and black discoloration on the surface of the tooth. Antimicrobial agents in whitening dentifrices might pass through the lipophilic bacterial membrane and cause disruption of the bacterial structure in the pellicle, thereby reducing extrinsic stains. Triclosan and methyl paraben are the most commonly used antimicrobial agents in whitening dentifrices (7).

Nathoo et al. (39) have compared the extrinsic stain removal efficacy of a dentifrice containing triclosan, PVM/MA copolymer, $\mathrm{NaF}$, and specially designed silica for sensitivity relief to a negative control dentifrice containing $\mathrm{NaF}$. They reported that the whitening effect of the dentifrice containing triclosan, PVM/ MA copolymer, and NaF on extrinsic stains was significantly more than that in the negative control group.

\section{Recent Developments in Whitening Dentifrices}

Charcoal is a good absorbent, and charcoal-containing preparations have been used primarily for acute poisoning and drug overdose (40).

The use of charcoal for oral hygiene started with Hippocrates in the Ancient Greek period (4I). Charcoal dust for tooth cleaning has been used in different ways, such as applying with finger on teeth, chewing sticks, or toothpaste (42).

Nowadays, many dentifrices containing active carbon or activated charcoal are manufactured and sold on internet sites and in the market for whitening purposes. Micro charcoal in dentifrices is claimed to absorb dirt and clean the teeth and gaps between the teeth that are difficult to reach (43). However, charcoal, has been explained as an abrasive mineral for the teeth or gingival tissues, and products containing charcoal have raised concerns about damage to these oral structures (44). A dentifrice containing charcoal, silica, and hydrated silica caused significant changes in the enamel surface roughness that exceeded the threshold of bacterial retention of $0.2 \mu \mathrm{m}$ after brushing for an equivalent of 3 months. Star-shaped or so-called fractal shaped charcoal particles and larger average size of particles of the charcoal dentifrice are believed to be responsible for the increased surface roughness (43).

In a study on oral hygiene habits that was performed in Malaysia, Yaacob and Park (45) found that $8.9 \%$ of the population using charcoal or table salt has varying degrees of severity of concave cavities into dentin formed on the labial surfaces of the teeth, resulting in the teeth becoming less white, even yellowish, with enamel loss.

Despite the reported theory that older types of charcoal-containing products are harmful, a manufacturer claims that activated carbon must not be confused with raw charcoal powder dentifrices because activated carbon is created by mixing charcoal powder into the dentifrice at the stage of manufacturing, creating an homogenous non-abrasive product that whitens teeth by absorbing stains; in contrast, the charcoal powder whitens teeth by rubbing particles against the teeth (46). It is also claimed that dentifrices that contain activated carbon have an ingredient that coats the tooth surface with a blue tint to influence light reflection and provide additional whitening (44).

Brooks et al. (42) examined 50 activated charcoal-containing dentifrices and found that these dentifrices have been introduced to the market with consumer-appealing terms, such as ecological, herbal, and organic labels. However, only 4 of the 50 examined dentifrices are reported to contained fluoride. Although $96 \%$ of these dentifrices claim to exert a whitening effect, to our knowledge, no controlled clinical studies have proven these claims. Thus, dentists should inform their patients about the unproven effects of these dentifrices and the consequences that may result from the lack of fluoride (42).

\section{Safety of Whitening Dentifrices}

It is important to ensure that any oral care product does not have any adverse effect on the tooth, dental materials, and soft tissues. There are some concerns about the adverse effects of ingredients of whitening dentifrices on dental hard and soft tissues.

In high concentrations, protease enzymes, such as papain and bromelain can theoretically cause irritation in the soft tissues. However, papain and bromelain in dentifrices have not been associated with adverse effects (7).

DeSalva et al. (47) have showed that triclosan in the dental products can be tolerated by the human body. However, continuous exposure to antimicrobial agents may raise microbial resistance and result in an inadequate immune response when virulent microorganisms are encountered (7). There are insufficient clinical studies regarding the long-time use of whitening dentifrices; therefore, dentists should consider the long-time adverse effects of triclosan.

Pyrophosphates in tartar control dentifrices and whitening dentifrices have been reported in only one study wherein they caused cheilit and circulum dermatitis in patients (48).

A sodium lauryl sulfate containing dentifrice has been compared with a non-sodium lauryl sulfate containing-dentifrice by Veys et al. (49). They observed hyperceratinized epithelial changes in the mucosae exposed to sodium lauryl sulfate for a long-time.

As per one study, a whitening dentifrice containing titanium dioxide caused Yellow Nail Syndrome. It is known that this syndrome manifests symptoms, such as yellow nails, lymphedema, 
and respiratory disorder, that are associated with titanium in adults (titanium dioxide-containing drugs, dental implants). Hsu et al. (50) reported the case of a 9-year-old patient with Yellow Nail syndrome who swallowed the dentifrice containing titanium dioxide during brushing. Thus, this syndrome was first noted in children and titanium dioxide-containing toothpastes are not recommended for use in children.

In conclusion, nowadays, increasing aesthetic concerns owing to the influence of the media have encouraged the use of easily accessible whitening dentifrices by patients and have made whitening technologies important for dentists. Despite a number of in vitro and in vivo studies on whitening dentifrices, there is no consensus on the best whitening technology with dentifrices. Thus, dentists should be aware of the contents of these dentifrices, recommend the appropriate onefor their patients, and warn about the possible abrasive effects.

Peer-review: Externally peer-reviewed.

Author contributions: Concept - C.D., A.M.; Design - A.M.; Supervision - A.M.; Resource - C.D.; Materials - C.D.; Data Collection and/or Processing - C.D.; Analysis and/or Interpretation - C.D., A.M.; Literature Search - C.D.; Writing - C.D., A.M.; Critical Reviews - A.M.

Conflict of Interest: Authors have no conflicts of interest to declare.

Financial Disclosure: The authors declared that this study has received no financial support.

\section{REFERENCES}

I. Joiner A. Whitening toothpastes: A review of the literature. J Dent 20I0; 38: el7-24. [Crossref]

2. Li Y. Stain removal and whitening by baking soda dentifrice: A review of literature. J Am Dent Assoc 2017; I48(IIS): S20-6. [Crossref]

4. Hasson $H$, Ismail A, Neiva G. Home-based chemically-induced whitening of teeth in adults. Cochrane Database Syst Rev 2006; (4): CD006202. [Crossref]

5. Demarco FF, Meireles SS, Masotti AS. Over-the-counter whitening agents: a concise review. Braz Oral Res 2009; 23: 64-70. [Crossref]

6. Pintado-Palomino K, Vasconcelos CVM, Silva RJ da, Fressatti AL de M, Motta BJG da, Pires-DE-Souza F de CP, et al. Effect of whitening dentifrices: a double-blind randomized controlled trial. Braz Oral Res 2016; 30(I): e82. [Crossref]

7. Sheen S, Pontefract H, Moran J. The benefits of toothpaste--real or imagined? The effectiveness of toothpaste in the control of plaque, gingivitis, periodontitis, calculus and oral malodour. Dent Update 200l; 28(3): |44-7. [Crossref]

8. White DJ. Development of an improved whitening dentifrice based upon "stain-specific soft silica" technology. J Clin Dent 200I; 12(2): 25-9.

9. Joiner A. The cleaning of teeth. In: Johansson I, Somasundaran P, editors. Handbook for cleaning/decontamination of surfaces - vol.I. Basel: Karger, 2007 p.37l-405 [Crossref]

10. Turker SB, Biskin T. Effect of three bleaching agents on the surface properties of three different esthetic restorative materials. J Prosthet Dent 2003; 89(5): 466-73. [Crossref]

II. Hefferren JJ. A laboratory method for assessment of dentrifrice abrasivity. J Dent Res 1976; 55(4): 563-73. [Crossref]

12. International Organizational for Standardization. (2017). Dentistry, dentifrices requirements, test methods and marking (ISO Standard No.Il609). Retrieved from https://www.iso.org/standard/70956.html

13. Wülknitz P. Cleaning power and abrasivity of European toothpastes. Adv Dent Res 1997; II(4): 576-9. [Crossref]

14. Lippert F. An introduction to toothpaste - its purpose, history and ingredients. Monogr Oral Sci 2013; 23: I-14. [Crossref]
15. Rice DE, Dhabhar DJ, White DJ. Laboratory stain removal and abrasion characteristics of a dentifrice based upon a novel silica technology. J Clin Dent 200I; I2(2): 34-7.

16. Singh S, Mankodi S, Chaknis P, Petrone ME, DeVizio W, Volpe AR, et al. The clinical efficacy of a new tooth whitening dentifrice formulation: a six-month study in adults. J Clin Dent 2002; 13(2): 8690.

17. Ayad F, Khalaf A, Chaknis P, Petrone ME, DeVizio W, Volpe AR, et al. Clinical efficacy of a new tooth whitening dentifrice. J Clin Dent 2002; 13(2): 82-5.

18. Joiner A, Pickles MJ, Matheson JR, Weader E, Noblet L, Huntington E. Whitening toothpastes: effects on tooth stain and enamel. Int Dent J 2002; 52: 424-30. [Crossref]

19. Hannig $M$, Joiner $A$. The structure, function and properties of the acquired pellicle. Monogr Oral Sci 2006; 19: 29-64. [Crossref]

20. Koertge TE, Brooks CN, Sarbin AG, Powers D, Gunsolley JC. A longitudinal comparison of tooth whitening resulting from dentifrice use. J Clin Dent 1998; 9(3): 67-71.

21. Yankell SL, Emling RC, Petrone ME, Rustogi K, Volpe AR, DeVizio W, et al. A six-week clinical efficacy study of four commercially available dentifrices for the removal of extrinsic tooth stain. J Clin Dent 1999; 10: 115-8.

22. Bollmer BW, Sturzenberger OP, Vick $\vee$, Grossman E. Reduction of calculus and peridex stain with tartar-control crest. J Clin Dent 1995; 6(4): 185-7.

23. Hoic D, Dixit N, Prencipe M, Subramanyam R, Cameron R, Abdel Malak R, et al. The technology behind Colgate Simply White Toothpaste. J Clin Dent 2004; 15(2): 37-40.

24. Sharma N, Galustians HJ, Qaqish J, Rustogi K, Zhang YP, Petrone $\mathrm{ME}$, et al. Comparative tooth whitening and extrinsic tooth stain prevention efficacy of a new dentifrice and a commercially available tooth whitening dentifrice: six-week clinical trial. J Clin Dent 2004; 15(2): 52-7.

25. Kleber CJ, Putt MS, Nelson BJ. In vitro tooth whitening by a sodium bicarbonate/peroxide dentifrice. J Clin Dent 1998; 9(I): 16-21.

26. Soares CNGS, Amaral FLB do, Mesquita MF, Franca FMG, Basting RT, Turssi CP. Toothpastes containing abrasive and chemical whitening agents: efficacy in reducing extrinsic dental staining. Gen Dent 2015; 63(6): e24-8.

27. Shellis RP, Addy M, Rees GD. In vitro studies on the effect of sodium tripolyphosphate on the interactions of stain and salivary protein with hydroxyapatite. J Dent 2005; 33(4): 313-24. [Crossref]

28. Baig A, He T, Buisson J, Sagel L, Suszcynsky-Meister E, White DJ. Extrinsic whitening effects of sodium hexametaphosphate--a review including a dentifrice with stabilized stannous fluoride. Compend Contin Educ Dent 2005; 26(9 Suppl I): 47-53.

29. Patil PA, Ankola AV, Hebbal MI, Patil AC. Comparison of effectiveness of abrasive and enzymatic action of whitening toothpastes in removal of extrinsic stains - a clinical trial. International J Dent Hyg 2015; I3(I): 25-9. [Crossref]

30. Feijoo-Siota L, Villa TG. Native and biotechnologically engineered plant proteases with industrial applications. Food Bioprocess Technol 20ll; 4: 1066-88. [Crossref]

31. Chakravarthy P, Acharya S. Efficacy of extrinsic stain removal by novel dentifrice containing papain and bromelain extracts. J Young Pharm 2012; 4(4): 245-9. [Crossref]

32. Lopes MC, Mascarini RC, da Silva BMCG, Flório FM, Basting RT. Effect of a papain-based gel for chemomechanical caries removal on dentin shear bond strength. J Dent Child (Chic) 2007; 74(2): 93-7.

33. Kalyana P, Shashidhar A, Meghashyam B, SreeVidya KR, Sweta S. Stain removal efficacy of a novel dentifrice containing papain and Bromelain extracts - an in vitro study. Int J Dent Hyg 20Il; 9(3): 22933. [Crossref]

34. Lyon TC, Parker WA, Barnes GP. Evaluation of effects of application of a citroxain-containing dentifrice. J Esthet Dent 1991; 3(2): 5I-3. [Crossref] 
35. CIE, Colorimetry, (3rd edition), CIE, Publication, Central Bureau of the CIE (2004).

36. Joiner A, Luo W. Tooth colour and whiteness: A review. J Dent 2017; 67: S3-10. [Crossref]

37. Collins LZ, Naeeni M, Platten SM. Instant tooth whitening from a silica toothpaste containing blue covarine. J Dent 2008; 36: 2I-5. [Crossref]

38. Haywood VB. Achieving, maintaining, and recovering successful tooth bleaching. J Esthet Dent 1996; 8(I): 31-8. [Crossref]

39. Nathoo S, Mateo LR, Delgado E, Zhang YP, DeVizio W. Extrinsic stain removal efficacy of a new dentifrice containing $0.3 \%$ triclosan, 2.0\% PVM/MA copolymer, $0.243 \%$ NaF and specially-designed silica for sensitivity relief and whitening benefits as compared to a dentifrice containing 0.3\% triclosan, 2\% PVM/MA copolymer, $0.243 \% \mathrm{NaF}$ and to a negative control dentifrice containing 0.243\% NaF: a 6-week study. Am J Dent 20Il; 24 Spec No A: 28A-3IA.

40. Juurlink DN. Activated charcoal for acute overdose: a reappraisal. $\mathrm{Br} J$ Clin Pharmacol 2016; 8I(3): 482-7. [Crossref]

41. Newsom SWB. Hygiene and the ancient Romans. J Infect 2004; 5: 25-7. [Crossref]

42. Brooks JK, Bashirelahi N, Reynolds MA. Charcoal and charcoal-based dentifrices: A literature review. J Am Dent Assoc 2017; 148(9): 661-70. [Crossref]
43. Pertiwi UI, Eriwati YK, Irawan B. Surface changes of enamel after brushing with charcoal toothpaste. J Phys Conf Ser 2017; 884: 0I2002. [Crossref]

44. DiGangi P. Black toothpaste and white teeth: When opposites collide pearls for practice. https://www.dentistryiq.com/articles/2017/03/black-toothpaste-and-white-teeth-when-opposites-collide.html.

45. Yaacob HB, Park AW. Dental abrasion pattern in a selected group of Malaysians. J Nihon Univ Sch Dent 1990; 32(3): 175-80. [Crossref]

46. Curaprox "Black is White", New gold standard activated carbon toothpaste, launches in Canada. Oral Health Group. hHps://www. oralhealthgroup.com/products/curaprox-black-is-white-new-goldstandard-activated-carbon-toothpaste-launches-in-canada/.

47. DeSalva SJ, Kong BM, Lin YJ. Triclosan: a safety profile. Am J Dent 1989; 2 Spec No: 185-96.

48. Beacham BE, Kurgansky D, Gould WM. Circumoral dermatitis and cheilitis caused by tartar control dentifrices. J Am Acad Dermatol 1990; 22(6 Pt I): 1029-32. [Crossref]

49. Veys RJ, Baert JH, De Boever JA. Histological changes in the hamster cheek pouch epithelium induced by topical application of sodium lauryl sulphate. Int J Exp Pathol 1994; 75(3): 203-9.

50. Hsu T-Y, Lin C-C, Lee M-D, Chang BP-H, Tsai J-D. Titanium Dioxide in toothpaste causing yellow nail syndrome. Pediatrics 2017; 139: e20160546. [Crossref] 